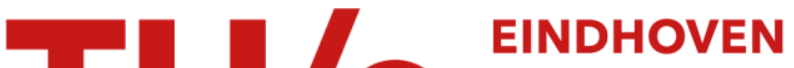

\section{Fast and robust method for measuring semiconductor optical amplifier gain}

\section{Citation for published version (APA):}

Pustakhod, D., Williams, K., \& Leijtens, X. (2018). Fast and robust method for measuring semiconductor optical amplifier gain. IEEE Journal of Selected Topics in Quantum Electronics, 24(1), [8004439].

https://doi.org/10.1109/JSTQE.2017.2737581

DOI:

10.1109/JSTQE.2017.2737581

Document status and date:

Published: 01/01/2018

\section{Document Version:}

Publisher's PDF, also known as Version of Record (includes final page, issue and volume numbers)

\section{Please check the document version of this publication:}

- A submitted manuscript is the version of the article upon submission and before peer-review. There can be important differences between the submitted version and the official published version of record. People interested in the research are advised to contact the author for the final version of the publication, or visit the $\mathrm{DOI}$ to the publisher's website.

- The final author version and the galley proof are versions of the publication after peer review.

- The final published version features the final layout of the paper including the volume, issue and page numbers.

Link to publication

\section{General rights}

Copyright and moral rights for the publications made accessible in the public portal are retained by the authors and/or other copyright owners and it is a condition of accessing publications that users recognise and abide by the legal requirements associated with these rights.

- Users may download and print one copy of any publication from the public portal for the purpose of private study or research.

- You may not further distribute the material or use it for any profit-making activity or commercial gain

- You may freely distribute the URL identifying the publication in the public portal.

If the publication is distributed under the terms of Article 25fa of the Dutch Copyright Act, indicated by the "Taverne" license above, please follow below link for the End User Agreement:

www.tue.nl/taverne

Take down policy

If you believe that this document breaches copyright please contact us at:

openaccess@tue.nl

providing details and we will investigate your claim. 


\title{
Fast and Robust Method for Measuring Semiconductor Optical Amplifier Gain
}

\author{
Dzmitry Pustakhod, Kevin Williams, and Xaveer Leijtens, Senior Member, IEEE
}

\begin{abstract}
In this paper, we present a new, robust multipoint fitting method for gain measurement with a metric for quality estimation of the procedure. The method is able to identify the deleterious effect of imperfections within the test structures, is tolerant to optical coupling errors and is well suited to high throughput, generic, automated testing of semiconductor optical amplifiers. Gain is estimated in a range of pump current densities over multiple spectral bands from 1400 to $1600 \mathrm{~nm}$ with a standard error in the order of $1 / \mathrm{cm}$.
\end{abstract}

Index Terms-Gain measurement, semiconductor optical amplifiers, photonic integrated circuits, segmented device, active-passive integration.

\section{INTRODUCTION}

$\mathbf{S}$ EMICONDUCTOR optical amplifiers (SOAs) play an important role as a component in photonic integrated circuits. They serves as a light source and as an amplifier and form the gain section of various lasers [1]. Fast and reliable methods for measuring SOA parameters, in particular, its gain, are needed to characterize SOA performance for accurate circuit design and process optimisation.

Several methods to measure gain versus wavelength of integrated semiconductor optical amplifiers have been proposed. They include transmission-based methods [2], the Hakki-Paoli method and spectral-fringe-based methods [3]-[9] based on analysis of an amplified spontaneous emission (ASE) spectrum in a Fabry-Pérot (FP) cavity. A number of additional methods are based on the comparison of different SOA lengths [10][15]. These methods rely on assumptions about the facet quality, variations between structures and optical coupling and do not provide any information about the quality of the measurement. Moreover, they can require using a high resolution optical spectrum analyser, precision alignment and in the case of variable length SOAs, repeated optical coupling.

In this paper, we present a fast and robust method for SOA gain measurement based on the multi-point fitting of measurements from a single multi-section device (Fig. 1). The

Manuscript received April 10, 2017; revised June 8, 2017; accepted August 1, 2017. Date of publication August 8, 2017; date of current version August 29 , 2017. This work was carried out in the ProCon Project 11369, supported by the Dutch Technology Foundation STW, which is part of the Netherlands Organization for Scientific Research (NWO), and which is funded in part by the Dutch Ministry of Economic Affairs. (Corresponding author: Dzmitry Pustakhod.)

The authors are with the COBRA Research Institute, Eindhoven University of Technology, Eindhoven $5600 \mathrm{MB}$, The Netherlands (e-mail: d.pustakhod@tue.nl; K.A.Williams@tue.nl; x.j.m.leijtens@tue.nl).

Color versions of one or more of the figures in this paper are available online at http://ieeexplore.ieee.org.

Digital Object Identifier 10.1109/JSTQE.2017.2737581

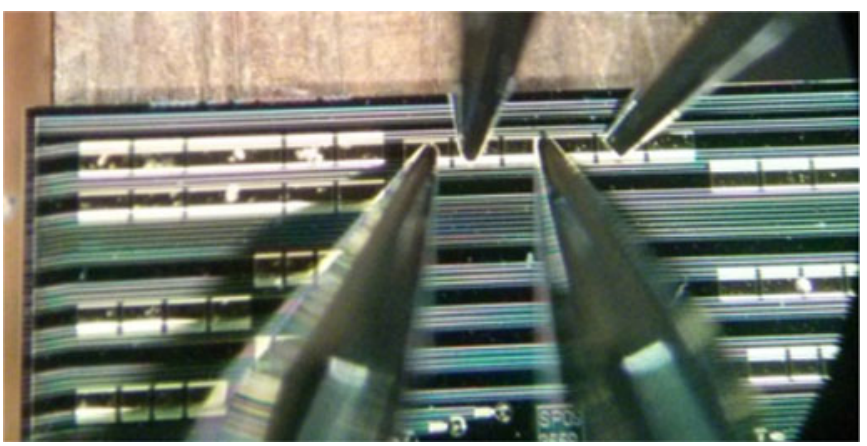

Fig. 1. Micrograph of the chip with test structures manufactured by smart photonics.

measurement requires only one optical coupling, and provides a metric for quality control of the measurement procedure. Using the proposed method, we show that measurement errors can be identified when using test structures with absorbing electrical isolation, motivating the use of optically passive waveguides between amplifiers sections.

A fabricated chip with test structures mounted on the measurement setup is shown in Fig. 1. The test cell shows an example multi-section SOA at the top of the chip with four electrical probes and just one optical connection at the left facet. Through an appropriate combination of measurements, the gain may be identified as a function of wavelength and current, with an indication of the quality of fit.

This paper is organised as follows. In Section II we give an overview of the current methods for SOA gain measurement. This is followed in Section III by a detailed description of variable SOA length methods for evaluating gain, including the structures proposed to implement it and the ways to calculate gain from the measured ASE spectra. In Section IV we study the sensitivity to coupling loss variations of different test structures, which implement a set of SOAs of different length, and identify those with tolerance to the coupling efficiency variation. Section V covers details of the test structure design and fabrication. Sections VI and VII describe the measurements we have performed and their results. The paper is completed with conclusions in Section VIII.

\section{Methods OvervieW}

The most intuitive approach to measure SOA gain is to scan a single wavelength signal and to measure the difference between input and output levels. More sophisticated, high-throughput 
transmission methods [2] measure gain directly over a large spectral range with spectrally broad laser probe light and require use of femtosecond pulsed laser. The measurement however combines unknown coupling losses and gain from the amplifier.

For amplifiers in Fabry-Pérot cavities several other methods can be used. The threshold current for a set of devices of different cavity length can be evaluated [16]. If the total optical loss in the cavity is known for each length, the plot of optical loss versus threshold current gives a relation of peak gain and current density.

A group of methods based on the analysis of the amplified spontaneous emission (ASE) spectrum in a Fabry-Pérot (FP) cavity has been studied intensively in the literature. In the method proposed by Hakki and Paoli [3], a spectral gain distribution is obtained from the peak-to-valley ratio of each cavity mode of the ASE spectrum. The measured peak height is sensitive to the resolution of the optical spectrum analyzer and therefore the Hakki-Paoli method may underestimate the gain value at current densities approaching the threshold current [8]. Cassidy et al. [4] have proposed using the ratio of the integral (mode sum) of the wavelength resolved power over one mode to the minimum power (instead of the peak-to-valley ratio in the Hakki-Paoli method) to derive the gain from the ASE spectrum. This method is much less sensitive to the response function of the optical spectrum analyzer (OSA) than the Hakki-Paoli method.

The Hakki-Paoli and Cassidy methods are sensitive to the noise as a result of the small intensity at the ASE spectrum minima. An improvement to reduce the sensitivity to the noise was made by Wang [5], who fitted the profile of each of the FP modes to an Airy function, thus obtaining a more accurate power value in the minimum. A method based on least square fitting was proposed by Zhang [6] to eliminate the error due to the asymmetric shape of longitudinal modes in a broader wavelength range. The noise limit problem is also addressed in the Fourier transform method and the Fourier series expansion (FSE) method [7]-[9], which calculate the gain based on the ratio of the intensity of the wavenumber-dependent DC- and first order AC-component in the transformed domain. They have a reduced noise sensitivity, as they use weighted integrals over the mode and not a single point measurement so that the noise is partly reduced by summation.

Methods for gain measurement for single electrode amplifiers have so far been sensitive either to coupling variations, or measurement equipment resolution and noise floor limit due to the small light intensity at the minimum. This has lead researchers to study multiple electrode methods. They make use of the relation between output ASE spectrum intensity and the SOA length, as proposed by Oster in the variable stripe length method [10], [11]. Thomson et al. [12] have shown that for a set of two spectra for SOAs of different lengths, the modal gain values can be obtained at every wavelength. The drawback of these variable amplifier length methods is that a time-consuming and precision-optical alignment is needed for measuring ASE from each of the stripes. To guarantee a good accuracy of the methods it is also critical to have same coupling loss for all the structures, which is hard to control due to variations in the fabrication of (a)

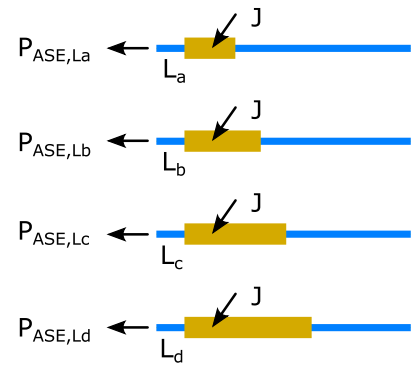

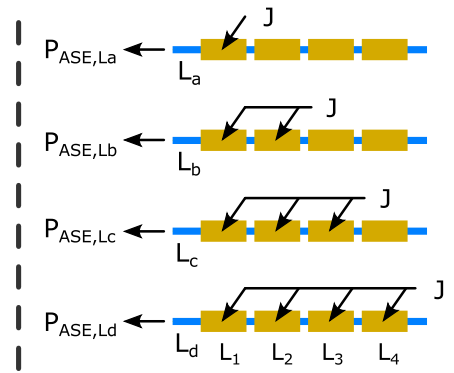

(b)
Fig. 2. Two types of test structures to implement variable amplifier length method: (a)—variable stripe length structures. A set of structures of different length is used. (b) — segmented device. Same structure is used, the actual length is varied by varying the applied currents. $L_{a}, \ldots, L_{d}$ are total emitter lengths, $L_{1}, \ldots, L_{4}$ are SOA section lengths.

optical waveguides. This can be solved at the expense of space and measurement time by increasing the number of stripes used and fitting the obtained result.

As an alternative to the set of devices of different length, sectioned devices with independent control of injection were proposed both for gain and absorption measurements [13]-[15]. This approach was further developed by integrating the light source and integrated spectrometer [17] to allow all-electrical gain measurement on wafer scale.

\section{VARIABLE AMPLIFIER LENGTH METHODS}

\section{A. Amplified Spontaneous Emission Versus amplifier Length}

The increase of the light intensity $P$ in an SOA is determined by the gain $g$ and the output spontaneous emission $P_{\mathrm{sp}}$ of the amplifier (both per unit length) [18]:

$$
\frac{d P}{d x}=g P+p_{\mathrm{sp}},
$$

where $P$ is light intensity in a given point within an amplifier. Gain here is the net modal gain. Provided we have no incoming light $\left(P(x=0)=P_{\text {in }}=0\right)$, integrating (1) for an SOA of length $L$, we calculate the measured single-pass amplified spontaneous emission intensity $P_{\mathrm{ASE}, \mathrm{L}}$ as [10]

$$
P_{\mathrm{ASE}, \mathrm{L}}(\lambda)=\eta_{\mathrm{c}} \frac{p_{\mathrm{sp}}(\lambda)}{g(\lambda)}\left(e^{g(\lambda) L}-1\right),
$$

where $\eta_{c}$ takes into account the output power coupling efficiency from the waveguide to the measurement equipment. Here, all the quantities have a wavelength dependency, therefore from (2), gain spectrum $g(\lambda)$ can be calculated from measured $P_{\mathrm{ASE}, \mathrm{L}}(\lambda)$ from SOAs of different length.

\section{B. Test Structures}

Two types of structures were used in the literature to realize a set of SOAs with different length: variable stripe length and segmented devices. Fig. 2 shows the difference between measurement procedure of these two types. The variable stripe length method [10], [11] employs multiple structures, each having a different optical output to be coupled to the measurement 
system. The measurement procedure consists of consecutive alignment to structures $a, b, c \ldots$ etc., and measurement of ASE spectra emitted by each of them (Fig. 2(a)).

A segmented contact structure [13]-[15] represents the second type of structures. It contains electrically isolated amplifier segments, which can be independently driven with an electrical current. During the measurement, light is always collected from the same output waveguide, and the effective amplifier length is varied by applying different combinations of pump current (Fig. 2(b)).

\section{Gain Calculation}

The gain spectrum can be calculated from a set of ASE spectra in several different ways. In this section we list the approaches which are discussed in the literature and will be compared in the present paper. Note, that the calculation procedure can be applied to the measurement results from both types of the structures described in Section III-B. This means that the same formulas can be applied to ASE spectra obtained from both variable-stripe-type or segmented-contact structures We consider two methods outlined by Thomson and Xin and our new multipoint fitting method.

1) Two-Point Thomson Method: Having two SOAs $a$ and $b$, one being twice as long as the other $L_{a}=L$ and $L_{b}=$ $2 L$, the gain can be calculated directly by using Thomson's approach [12]:

$$
g(\lambda)=\frac{1}{L} \ln \left(\frac{P_{\mathrm{ASE}, 2 \mathrm{~L}}(\lambda)}{P_{\mathrm{ASE}, \mathrm{L}}(\lambda)}-1\right) .
$$

2) Three-Point Xin Method: In the above approach any unguided spontaneous emission is not taken into account. Among the reasons of unguided emission could be the leakage current or emission into unguided waveguide modes [19]. The presence of unguided emission can lead to a systematic error in the gain calculation, and it should be taken care of at low pump current densities and gain values [19]. During measurements, the unguided radiation can be filtered out spatially when collecting light from the waveguide. A method proposed in [15] considers the unguided spontaneous emission radiation intensity $P_{\text {unguided }}$ as an addition to the amplified spontaneous emission from (2):

$$
P_{\mathrm{L}}=P_{\mathrm{ASE}, \mathrm{L}}+P_{\text {unguided }} .
$$

The unguided radiation can be eliminated in the gain calculation by comparing ASE spectra from SOA of three different lengths. SOA lengths should satisfy the following conditions: $L_{a}=L_{1}, L_{b}=L_{1}+L_{2}$, and $L_{c}=L_{1}+2 L_{2}$. The gain is then calculated as ${ }^{1}$

$$
g=\frac{1}{L} \ln \left(\frac{P_{\mathrm{L}_{1}+2 \mathrm{~L}_{2}}-P_{\mathrm{L}_{1}}}{P_{\mathrm{L}_{1}+\mathrm{L}_{2}}-P_{\mathrm{L}_{1}}}-1\right) .
$$

The disadvantage of above-mentioned methods is that if any of the measured values $P_{\mathrm{L}}$ contains an error, it will propagate to

\footnotetext{
${ }^{1}$ In [15], $L_{1}=L_{2}=L$, therefore the SOA lengths used are $L_{1}=L, L_{1}+$ $L_{2}=2 L$, and $L_{1}+2 L_{2}=3 L$. It is easy to show that this is not necessary, and (5) also holds for $L_{1} \neq L_{2}$
}

the calculated gain value and there is no means to discover the error.

3) Multipoint Fitting Nethod With Quality of Fit: We propose to extract the gain by using more available SOA lengths $L_{a}, L_{b}, \ldots$ and corresponding ASE values $P_{\mathrm{ASE}, \mathrm{L}_{\mathrm{i}}}$ and fitting them to either (2) or (4) by executing the following steps:

1) At each wavelength $\lambda$ we have a set of power densities $P_{i}$ measured from the SOAs of length $L_{i}$.

2) Calculate the measurement error for each of the data points. The error is determined by the limit of the OSA sensitivity $P_{\text {noise }}$ and measurement uncertainty $\epsilon$ : $\sigma_{i}=P_{\text {noise }}+\epsilon P_{i}$.

3) Calculate weight for each data point, which is inverse of the total measurement error: $w_{i}=1 / \sigma_{i}$

4) Perform weighted non-linear least-square fit of the data points, using (2) or (4) as a model function.

5) As a result of fitting, for every fit parameter we obtain its magnitude and standard error.

The steps described above are repeated for every wavelength.

The fitting approach has an intrinsic indication of the fitting quality, namely the standard error, which is calculated based on the residual sum of squares. It can be used to discard poor measurement results, e.g. due to a damaged waveguide in the middle of test structure or varying light collection efficiency.

In our paper we compare three mentioned methods: Thomson's (3), Xin's (5), and fitting to (2) and (4).

\section{SEnsitivity to Coupling Variation}

The calculation methods described in III-C commonly assume that the coupling efficiency $\eta_{\mathrm{c}}$ is the same for each measurement condition. The use of one output waveguide removes the need for structure-to-structure realignments, but the coupling efficiency is still affected for example by the chip displacement due to the height variations in a thermoelectric cooler for a temperature stabilised measurement. In this work we perform active realignment to the best coupling position after every pump current setting.

In the following section we perform a simulation to discover dependency of the test structure design, in particular, of SOA section lengths and their number, on the optical alignment induced error.

\section{A. Simulation Procedure}

In order to estimate the effect of coupling variation on the calculated gain, we performed a Monte-Carlo simulation of the measurement procedure, while varying the fiber location around optimum.

In the simulation we vary the relative offset of the fiber with respect to the center of the waveguide in the transverse and latelar directions. These two offsets are assumed to be independent and have a normal distribution with the mean value of zero. The random offset value gives a random variation of the coupling efficiency $\eta_{c}$ in (2). The standard deviation for the offsets is selected so that the coupling efficiency varies by less than $0.1 \mathrm{~dB}$. 


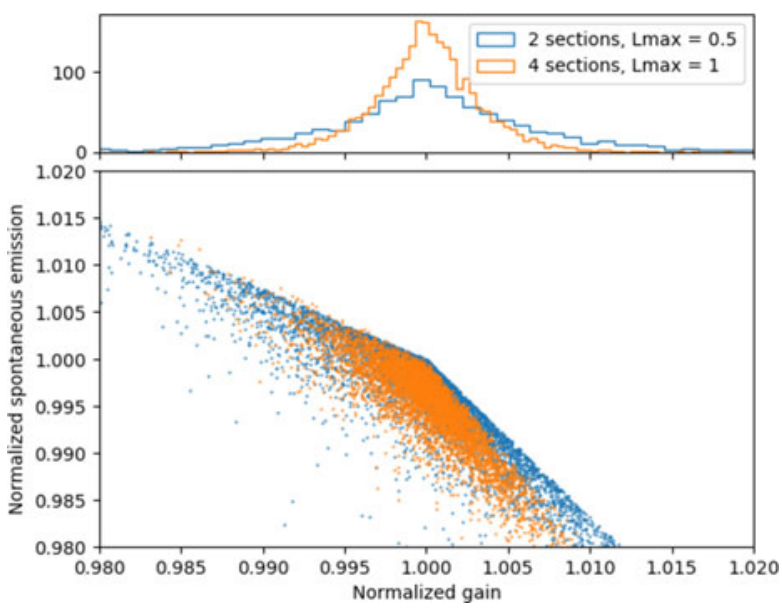

Fig. 3. Monte-Carlo simulation of the calculated gain distribution for two different designs of multi-section structures.

TABLE I SOA LENGTHS FOR MONTE-CARLo SimUlation

\begin{tabular}{lcc}
\hline \hline $\begin{array}{l}\text { \# SOA } \\
\text { lengths }\end{array}$ & $\begin{array}{c}\text { Maximum length, } \\
\max \left(L_{i}\right)\end{array}$ & $\begin{array}{c}\text { SOA lengths, } \\
L_{i}\end{array}$ \\
\hline 2 & 400 & 200,400 \\
2 & 600 & 300,600 \\
2 & 800 & 400,800 \\
3 & 300 & $100,200,300$ \\
3 & 450 & $150,300,450$ \\
3 & 600 & $200,400,600$ \\
3 & 800 & $266,533,800$ \\
3 & 400 & $100,200,400$ \\
3 & 600 & $150,300,600$ \\
3 & 800 & $200,400,800$ \\
4 & 400 & $100,200,300,400$ \\
4 & 600 & $150,300,450,600$ \\
4 & 800 & $200,400,600,800$ \\
6 & 600 & $100,200,300, \ldots, 600$ \\
6 & 800 & $133,266,400, \ldots, 800$ \\
10 & 800 & $700,710, \ldots, 790,800$ \\
\hline \hline
\end{tabular}

All lengths are in $\mu \mathrm{m}$.

The simulated ASE power values $P_{\mathrm{ASE}, \mathrm{L}_{\mathrm{i}}}$ are calculated at a single wavelength from (2) with typical nominal values of gain $g_{\mathrm{n}}$, spontaneous emission $p_{\mathrm{sp}, \mathrm{n}}$, and a set of lengths $L_{a}, L_{b}, \ldots$. From a set of ASE power values we calculate the gain $g_{\mathrm{s}}$ and spontaneous emission $p_{\mathrm{sp}, \mathrm{s}}$ using our fitting method described in the Section III-C3.

The deviations of the simulated values from the nominal values are represented as relative errors $\delta g=\left(g_{\mathrm{n}}-g_{\mathrm{s}}\right) / g_{\mathrm{n}}$ and $\delta p_{\mathrm{sp}}=\left(p_{\mathrm{sp}, \mathrm{n}}-p_{\mathrm{sp}, \mathrm{s}}\right) / p_{\mathrm{sp}, \mathrm{n}}$.

An example of the Monte-Carlo simulation result with 5000 trials is shown in Fig. 3. From this sample we plot a histogram and calculate the standard deviation $\sigma_{g}$ of the gain from the nominal value, which is an indicator of gain measurement precision for a specified misalignment.

\section{B. Simulation Results}

We carried out the Monte-Carlo simulation for different sets of SOA lengths varying the number of lengths and the maximum total length (Table I). In the table, the number of SOA lengths

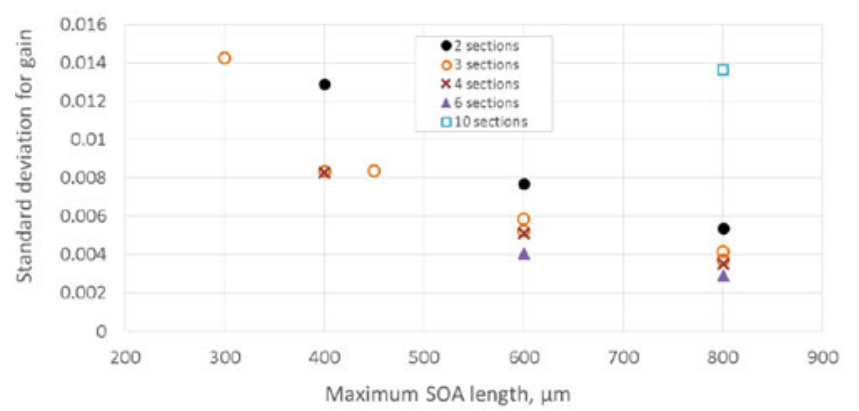

Fig. 4. Standard deviation of gain for sections of different length.

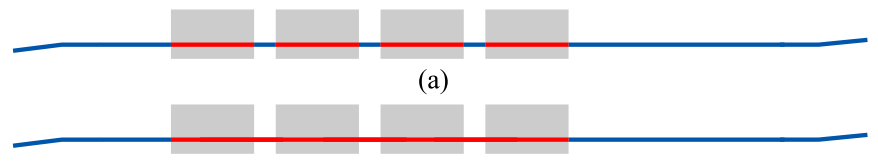

(b)

Fig. 5. Test structure schematic with (a) passive and (b) active isolations between SOAs.

determines the number of data points $P_{\mathrm{ASE}, \mathrm{L}_{\mathrm{i}}}$ used to calculate the gain.

The corresponding standard deviation for the gain is plotted in Fig. 4 as a function of the maximum length $\max \left(L_{i}\right)$. The standard deviation is a direct measure of the sensitivity of the measurement technique to alignment variations. Fig. 4 shows that test structures with relatively short sections lead to a higher spread in gain variation and therefore to a reduced accuracy. The structures with larger maximum length have a lower standard deviation of the gain. For a given maximum length, a clear improvement in precision is observed as the number of sections is increased.

However there is a number of factors that limit the maximum length and the number of sections. The maximum length in our case was limited by the lasing starting in the cavity formed by waveguide facets at a higher pump currents. Heat generated in the SOAs may also play a negative role by changing the gain profile of the amplifiers. ASE-induced gain saturation should also be considered and can be reduced when selecting the maximum section lengths [20]. A higher number of sections requires more electrical probes to be used in the measurement. For these reasons devices with 4 and 5 sections are selected for this study.

\section{DESIGN AND FABRICATION}

\section{A. Structure Design}

In our experiment we used the multi-section devices, consisting of a single waveguide with several SOA sections grown by active-passive integration. The schematic of the structures is shown in Fig. 5. The thin blue line denotes a waveguide, the red stripes denote the parts of the waveguide within an active region, and gray boxes represent the SOA electrical contact pads. The SOA sections are separated by the electrical isolation regions, which provide the gap between the SOA electrodes of $30 \mu \mathrm{m}$ and ensure electrical isolation between the SOA sections. This allows them to be pumped independently. The left side output 


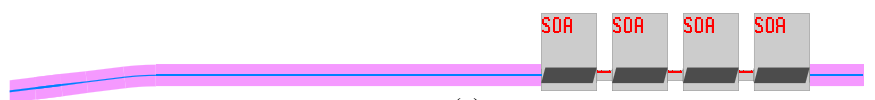

(a)

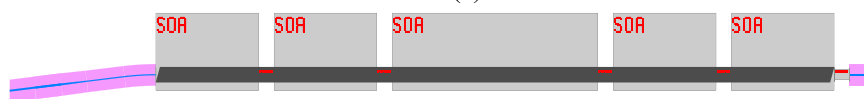

(b)

Fig. 6. Test structure mask designs with (a) passive and (b) active isolation sections. SOA lengths in $\mu \mathrm{m}$ : (a)—100, 100, 100, 100; (b)—200, 200, 400, 200, 200.

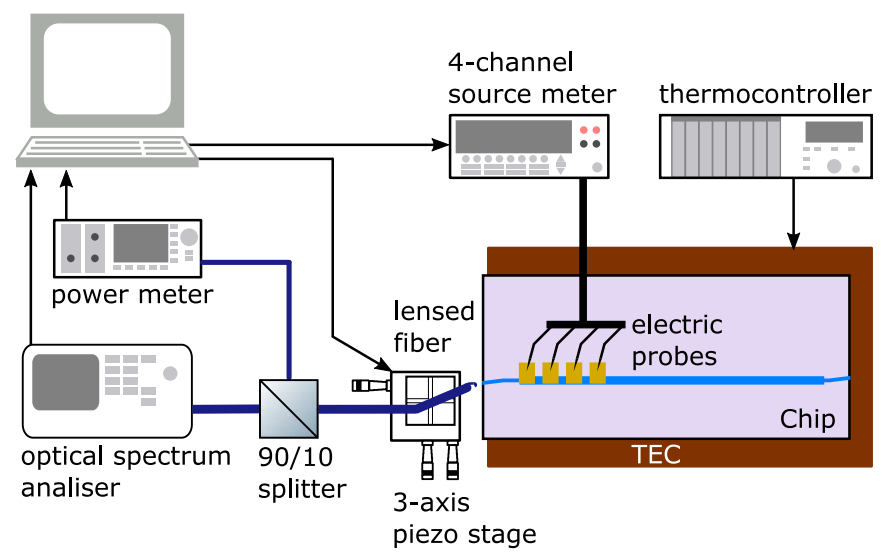

Fig. 7. Schematic of the measurement setup. TEC-thermoelectric cooler.

waveguides are angled at $7^{\circ}$ to suppress back reflection and suppress resonant light amplification.

In the paper we compare two structures. The first structure is depicted in Fig. 5(a). The absorption in the passive isolation sections between the amplifiers is small and can be neglected. The second structure is shown in Fig. 5(b). The isolation regions are made with the same active material as the amplifiers.

The mask designs of the actual structures is shown in Fig. 6 . Here, the black areas on top of the waveguides represent the active material location. First structure (Fig. 6(a)) contains 4 sections each having same length of $100 \mu \mathrm{m}$. The total SOA lengths $L_{i}$ achievable with it are therefore 100, 200, 300, and $400 \mu \mathrm{m}$. The second, all-active, structure (Fig. 6(b)) contains sections with lengths of 200, 200, 400, 200, and $200 \mu \mathrm{m}$.

\section{B. Fabrication}

InP-based test structures were fabricated by Smart Photonics in a multi-project wafer (MPW) run through the JePPIX.eu service [21]. The foundry provides active-passive integrated circuit, which allows lengths of active sections to be varied independently of the chip size. The structure design is realized with standard SOAs and isolation building blocks available from the design kit provided by the foundry.

\section{Measurement Setup}

In the measurement setup (Fig. 7), the chip is epoxy-bonded to a copper chuck to ensure electrical and thermal contact. In order to keep the chip temperature constant at different current densities, an active cooling by a thermo-electrical cooler (TEC) and a thermocontroller with a Thorlabs Pro8000 Mainframe is
TABLE II

MEASUREMENT CONFIGURATIONS

\begin{tabular}{lc}
\hline \hline $\begin{array}{l}\text { Signals applied to four } \\
\text { SOA sections }\end{array}$ & $\begin{array}{c}\text { Total length under } \\
\text { current injection, } \mu \mathrm{m}\end{array}$ \\
\hline$I, 0 \mathrm{~V}, 0 \mathrm{~V}, 0 \mathrm{~V}$ & $L_{a}=100$ \\
$I, I, 0 \mathrm{~V}, 0 \mathrm{~V}$ & $L_{b}=200$ \\
$I, I, I, 0 \mathrm{~V}$ & $L_{c}=300$ \\
$I, I, I, I$ & $L_{d}=400$ \\
\hline \hline
\end{tabular}

Current $I$ denotes a variable current which is proportional to the section length. $0 \mathrm{~V}$ is a short circuit.

used. Electrical signals from the source meters are provided through the individual electrical probes contacting the metal pads of the SOA sections.

A photograph of the chip installed on the measurement setup with the probe needles touching SOA contact pads is shown in Fig. 1.

Light emitted by the SOAs is collected through the chip facet with a single-mode lensed optical fiber. The fiber is mounted on the 3-axis piezo positioner, which allows accurate fiber alignment to the waveguide. An SOA emits in both TE and TM polarizations. We have measured the ratio of TE/TM amplified spontaneous emission intensity for a range of SOA lengths, which shows more than $10 \mathrm{~dB}$ difference between TE and TM gain and spontaneous emission over the $1500-1600 \mathrm{~nm}$ range. This translates into a systematic error not exceeding 5\%. A polarization filter may be used to separately measure TE and TM gain. To accommodate the temperature expansion of the mount and to keep coupling efficiency from the integrated waveguide to the lensed fiber constant, the fiber tip is realigned after each current setting. The collected light is then directed to an optical spectrum analyser ANDO AQ-6315A, which measures the resulting ASE spectrum with a resolution of $0.5 \mathrm{~nm}$. The OSA sensitivity was measured to be $P_{\text {noise }}=-90 \mathrm{dBm}$ and its measurement uncertainty is $\epsilon=0.3 \mathrm{~dB}$ from the specification.

\section{Measurement Results}

\section{A. ASE Spectra Measurement}

ASE is generated by the SOAs for a range of pump current densities $J$. In our experiments we covered the range from 0.25 to $10 \mathrm{kA} / \mathrm{cm}^{2}$.

Currents are applied to SOA sections in 4 different configurations of electrode bias (Table II). In the table, the sections are listed starting from the optical output side. The sections are either forward biased with a current $I$ or grounded $(0 \mathrm{~V})$. An example of measured ASE spectra from the structure in Fig. 5(a) at a current density $J=10 \mathrm{kA} / \mathrm{cm}^{2}$ is shown in Fig. 8. The spectra are for four different SOA lengths. This ensures four data points per wavelength for further gain calculation.

\section{B. Gain Calculation}

To compare our fitting method with other methods, we calculate the gain at each wavelength using four different approaches: 


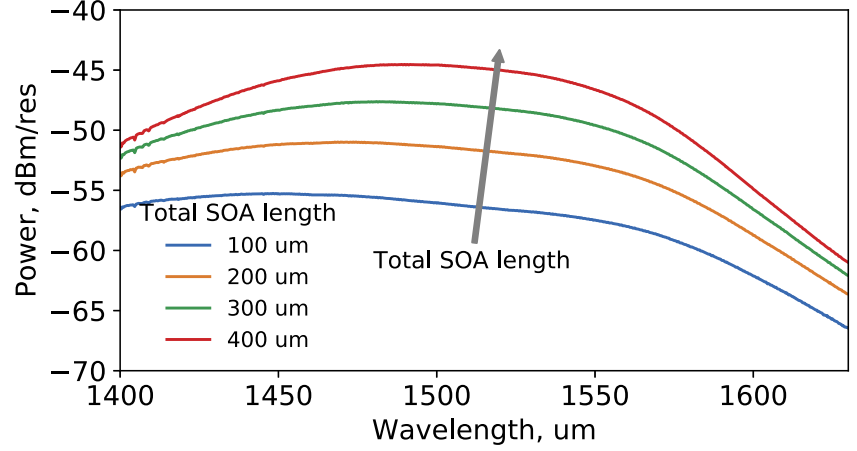

Fig. 8. Measured ASE for gain calculation for four different lengths. Pump current density is $J=10 \mathrm{kA} / \mathrm{cm}^{2}$ in each case.

i) Two-point Thompson method (3) from configurations $a$ and $b$. SOA lengths are $L=L_{a}=100 \mu \mathrm{m}$, and $2 L=$ $L_{b}=200 \mu \mathrm{m}$;

ii) Two-point Thompson method (3) from configurations $b$ and $d$. SOA lengths are $L=L_{b}=200 \mu \mathrm{m}$, and $2 L=$ $L_{d}=400 \mu \mathrm{m}$;

iii) Three-point Xin method (5) from configurations $a, b$ and $c$. SOA lengths are $L_{1}=L_{a}=100 \mu \mathrm{m}, L_{1}+L_{2}=$ $L_{b}=200 \mu \mathrm{m}$, and $L_{1}+2 L_{2}=L_{c}=300 \mu \mathrm{m}$;

iv) our method, four-point fitting to (2) from all configurations $a$ to $d$ are used. We followed the procedure described in the Section III-C3. An open-source Python package [22] was used to perform least-square fitting and calculate the standard error (SE).

Gain spectra have been derived from the ASE measurement data using the four methods as specified above, and these are compared in Fig. 9(a) for three different bias current densities. Net modal gain is defined as the optical gain $g$ in (1). On first inspection, the agreement over the $3 \mathrm{~dB}$ spectral width between the estimates is good, particularly for the $4.5 \mathrm{kA} / \mathrm{cm}^{2}$ bias point. However there are significant differences between the methods outside of the gain peak and at low and high values of current. Furthermore there is limited indication of the quality of the data.

We have no a-priori way to know which method gives the correct result. A commonly used method for measurement in the wide range of current densities is the two-point Thomson method, which we use as our reference method. However there are two length pairs ( $L=100,200 \mu \mathrm{m}$ and $L=200,400 \mu \mathrm{m})$ to which this method can be applied, since it is not known in advance which pair is best, our reference gain value is their mean value. The deviation of the gain calculated with the four methods from this reference value for the selected current density $J=$ $4.5 \mathrm{kA} / \mathrm{cm}^{2}$ is plotted as gain difference in Fig. 9(b). All four methods show a gain difference within $\pm 1 \mathrm{~cm}^{-1}$ within the top part of gain spectrum. The difference from the reference increases towards the edges of the wavelength range and its maximum value is within $\sim 7 \mathrm{~cm}^{-1}$.

Fig. 9(b) clearly shows the disagreement for the two-point Thompson measurements for the two different lengths pairs indicating a source of error even within the same test structure. Our multi-point fitting method gives values close to the reference value.

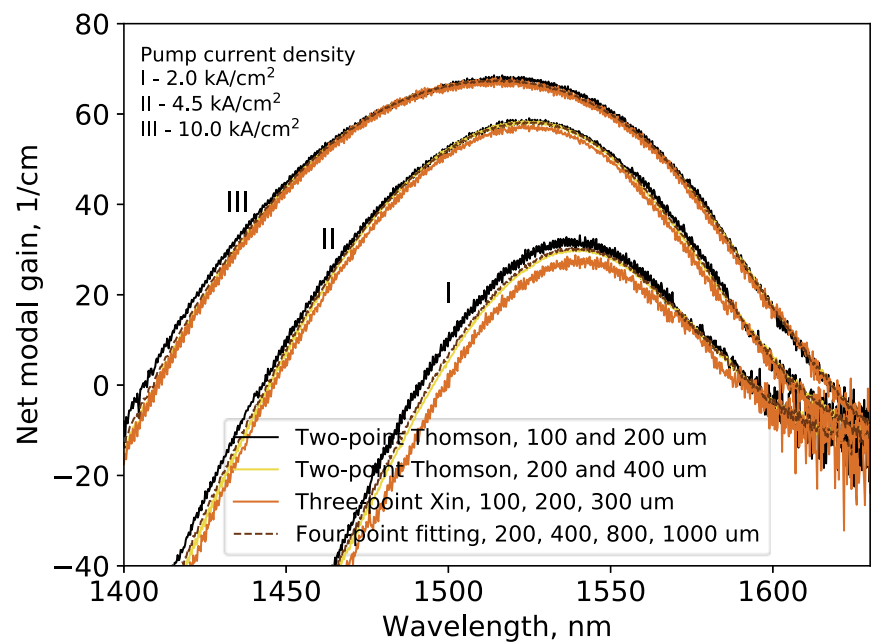

(a)

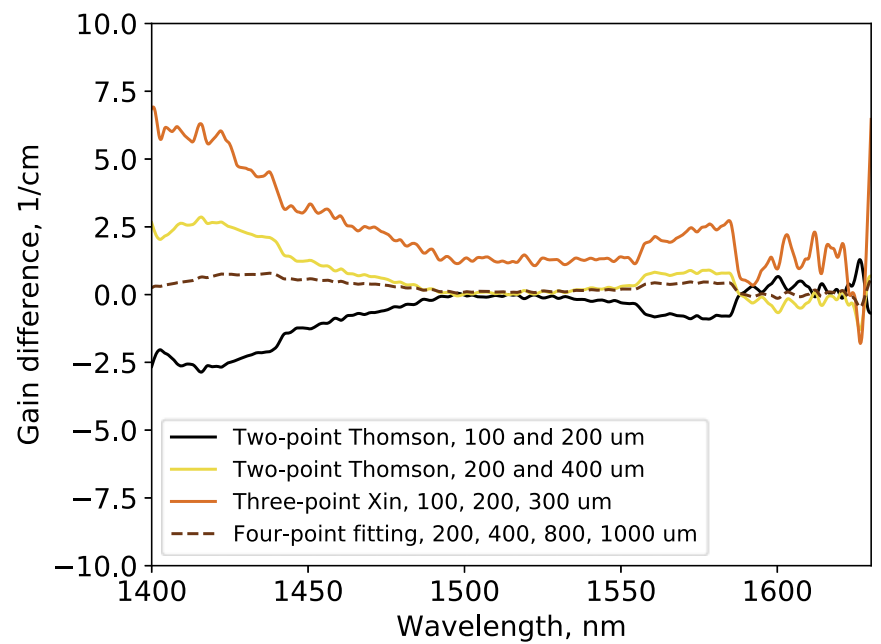

(b)

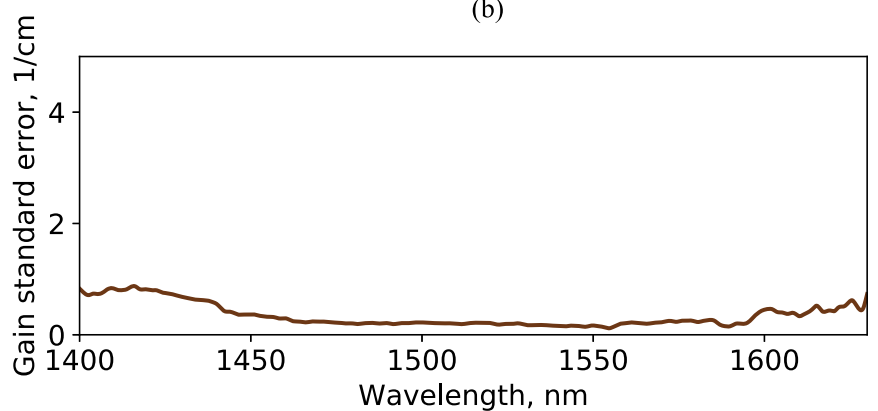

(c)

Fig. 9. Calculated net modal gain. (a) Comparison of gain calculated with different methods. (b) Difference of measured gain values from reference. Pump current density $J=4.5 \mathrm{kA} / \mathrm{cm}^{2}$. (c) Gain standard error obtained from the fourpoint fitting method.

We compare the maximum gain differences from the reference for a range of pump current densities in Fig. 10. For this figure, the maximum gain difference was specified over wavelength ranges with gain values above $-20 \mathrm{~cm}^{-1}$. The differences are particularly high for current densities below $2 \mathrm{kA} / \mathrm{cm}^{2}$, where spontaneous emission intensity and gain are low and ASE spectra reach the noise floor level of the OSA. The calculated gain therefore has a large spread, particularly at lower and higher 


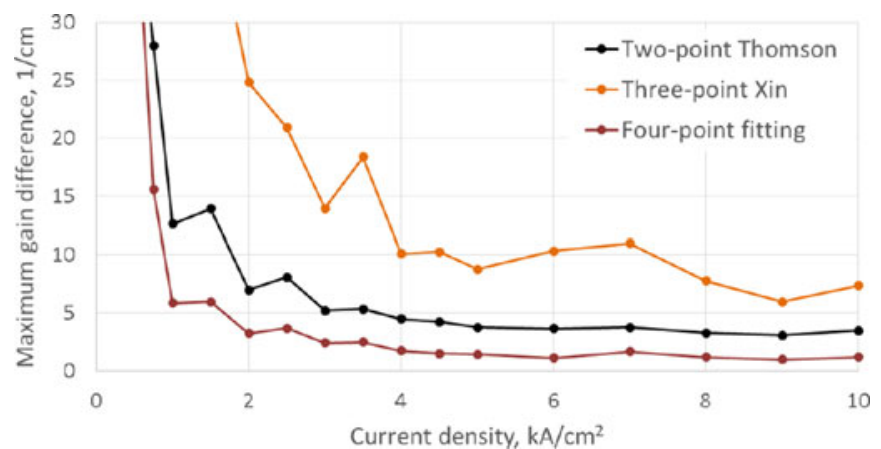

Fig. 10. Difference of calculated gain values from reference at different pump current densities.

wavelengths, similar to the variations shown in Fig. 9(a) above $1600 \mathrm{~nm}$. Fig. 10 shows that the four-point fitting method gives a much smaller variation from the reference value than two- and three-point analytical methods for the whole range of current densities.

\section{Quality of Fit}

Besides gain value, our fitting method allows confidence intervals to be calculated. Fig. 9(c) shows the standard error of the fitted gain, which is an important additional parameter obtained from the method. It is calculated automatically based on the residuals for each data point, and can be used for quality control of the method. This metric allows us to identify deviations from the amplified spontaneous emission model assumed in Section III-A. In case the structures are defective (due to design and/or fabrication), or, for example, the coupling was not constant during the measurements, this will show up through increased standard error values. Deviations from the approximation made in (1), such as the onset of laser oscillation or gain saturation will also be detected through this metric.

As a demonstration of this capability, we compare the two test structures described in Section V-A, one with optically passive isolation sections, and one with active isolation sections which may be expected to act as absorbing regions.

The data for the first structure with passive isolations are shown in the Fig. 9(a) and provide a control experiment. The derived gain spectra from the all-active with active isolation sections are shown in Fig. 11(a) for a current density $J=4.5 \mathrm{kA} / \mathrm{cm}^{2}$. Comparison of Figs. 11(a) and 9(a) shows that absorption at lower wavelengths leads to considerable difference in predicted gain for nominally the same amplifier building block design.

The standard error for the fitted gain for the second structure is shown in Fig. 11(b). It has a small value in the wavelength range above $1580 \mathrm{~nm}$, where the gain measurement is consistent with data in Fig. 9(a). However the standard error increased from $\sim 1 \mathrm{~cm}^{-1}$ in Fig. 9(c) to $\sim 5 \mathrm{~cm}^{-1}$ in Fig. 11(b), indicating unreliable data for the range $1420 \mathrm{~nm}$ to $1550 \mathrm{~nm}$ for the all-active structure. Absorption in the active isolation sections is expected to be insignificant at longer wavelengths, but can be expected to increase at lower wavelengths. The standard error metric indeed points out the problem in the measurement. By

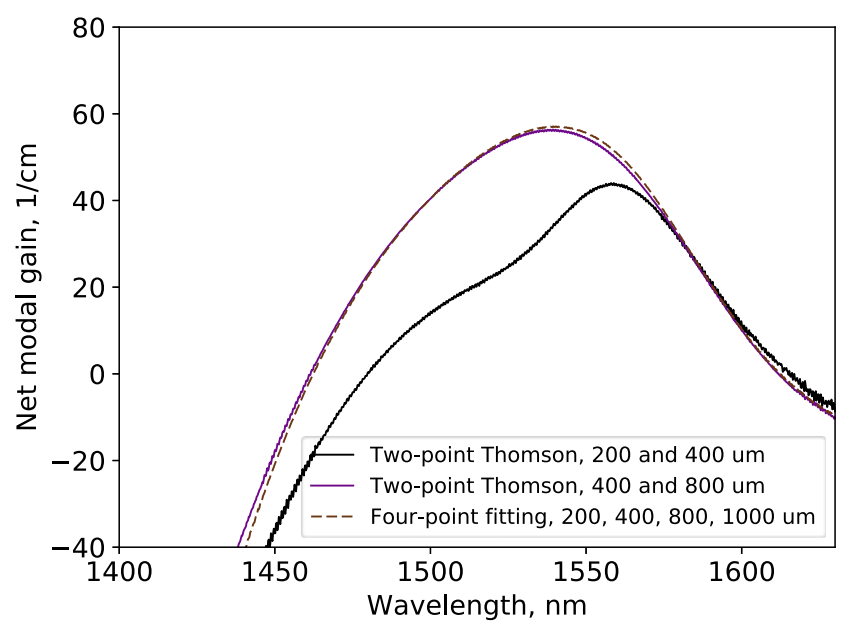

(a)

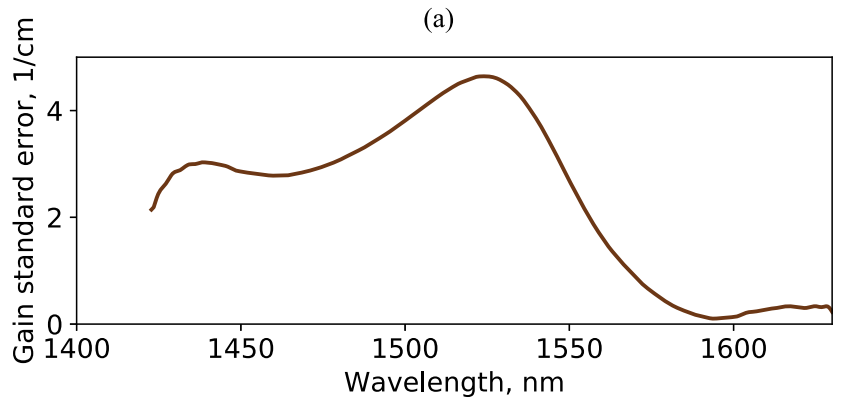

(b)

Fig. 11. Calculated net modal gain from the structure with isolation section containing active material. Pump current density is $J=4.5 \mathrm{kA} / \mathrm{cm}^{2}$. (a) Comparison of gain calculated with different methods. (b) Gain standard error obtained from the four-point fitting.

setting a threshold value for this metric, it is possible to include the quality control in the measurement procedure, which is important for automated testing

\section{Measurement Automation}

To measure the SOA net modal gain in the wide range of current densities, the described above measurements can be automated. We have applied the multi-point fitting method on the multi-section SOA test structure from Fig. 6(a) for current densities from 0.25 to $10 \mathrm{kA} / \mathrm{cm}^{2}$. The automated measurement procedure includes repetitive pump current setting with the particular configuration, fiber realignment, and spectrum measurement with OSA, followed by the gain calculation with a procedure described in Section III-C3. Fig. 12 shows the net modal gain obtained with the described procedure. The graph shows the expected blue shift of the peak gain value with the increased pump current density. The corresponding absolute standard error for the calculated gain is shown in Fig. 13. This parameter is below $1 \mathrm{~cm}^{-1}$ for the regions with positive gain, and increases towards the edges of the spectrum, particularly at lower current densities. This is due to the low intensity of spontaneous emission and low gain/absorption in the active material, which in total give the weak ASE intensity comparable to the sensitivity level of the OSA. Gain measurement at the extreme wavelengths can be improved by increasing the sensitivity of the OSA at the expense of the measurement 


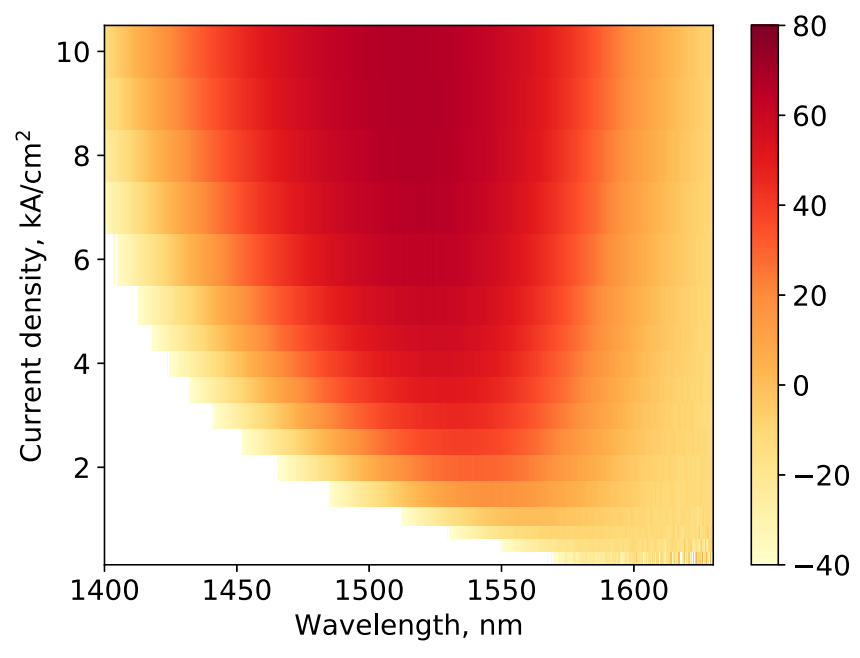

Fig. 12. Measured net modal gain spectrum in the range of pump current densities.

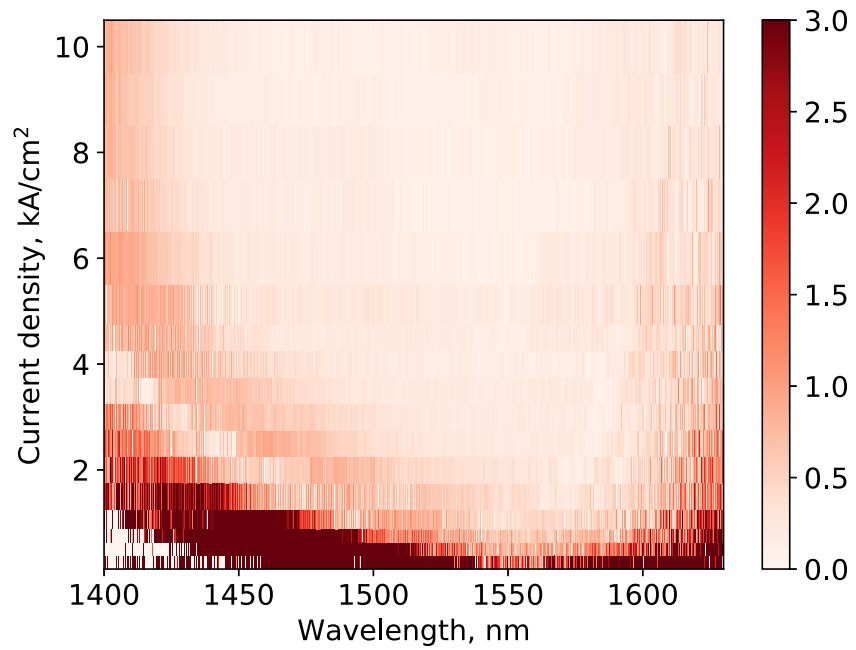

Fig. 13. Absolute standard error for the fitted net modal gain in the range of pump current densities.

time. This gives a high degree of confidence in the measurement data over the whole operating range of the SOA building block.

\section{CONCLUSION}

We presented a fitting method for calculating SOA gain based on multi-section devices. The method is more robust compared to other methods based on variable-length structures. We have shown that it provides a metric for quality estimation of the performed calculation, which is important for automation of the gain measurement. The technique is expected to be more resilient to optical alignment errors, and sensitive to imperfections such as undesired absorption in the test structure. The suitability for automation offers an attractive method for robust, high-throughput characterisation of optical amplifier building blocks for statistically more accurate data for design models and process optimisation.

\section{REFERENCES}

[1] M. Smit et al., "An introduction to InP-based generic integration technology," Semicond. Sci. Technol., vol. 29, no. 8, 2014, Art. no. 083001.

[2] C. Ellmers et al., "Measurement and calculation of gain spectra for (GaIn)As/(AlGa)As single quantum well lasers," Appl. Phys. Lett., vol. 72, no. 13, pp. 1647-1649, 1998. [Online]. Available: http://scitation. aip.org/content/aip/journal/ap1/72/13/10.1063/1.121140

[3] B. Hakki and T. Paoli, "CW degradation at $300{ }^{\circ} \mathrm{K}$ of GaAs doubleheterostructure junction lasers. II. Electronic gain," J. Appl. Phys., vol. 44 , no. 9, pp. 4113-4119, Sep. 1973.

[4] D. T. Cassidy, "Technique for measurement of the gain spectra of semiconductor diode lasers," J. Appl. Phys., vol. 56, no. 11, pp. 3096-3099, 1984

[5] H. Wang and D. T. Cassidy, "Gain measurements of Fabry-Peŕot semiconductor lasers using a nonlinear least-squares fitting method," IEEE $J$. Quantum Electron., vol. 41, no. 4, pp. 532-540, Apr. 2005.

[6] M. Zhang, D. Liu, and T. Makino, "Measurement of gain spectra of semiconductor lasers using least squares fitting method," IEEE Photon. Technol. Lett., vol. 25, no. 12, pp. 1122-1124, Jun. 2013.

[7] D. Hofstetter and J. Faist, "Measurement of semiconductor laser gain and dispersion curves utilizing Fourier transforms of the emission spectra," IEEE Photon. Technol. Lett., vol. 11, no. 11, pp. 1372-1374, Nov. 1999.

[8] W.-H. Guo, Y.-Z. Huang, C.-L. Han, and L.-J. Yu, "Measurement of gain spectrum for Fabry-Pérot semiconductor lasers by the fourier transform method with a deconvolution process," IEEE J. Quantum Electron., vol. 39, no. 6, pp. 716-721, Jun. 2003.

[9] W.-H. Guo, Q.-Y. Lu, Y.-Z. Huang, and L.-J. Yu, "Fourier series expansion method for gain measurement from amplified spontaneous emission spectra of Fabry-Pérot semiconductor lasers," IEEE J. Quantum Electron. vol. 40, no. 2, pp. 123-129, Feb. 2004.

[10] A. Oster, G. Erbert, and H. Wenzel, "Gain spectra measurements by a variable stripe length method with current injection," Electron. Lett., vol. 33, no. 10 , pp. 864-866, 1997.

[11] B. W. Tilma et al., "Measurement and analysis of optical gain spectra in 1.6 to $1.8 \mu \mathrm{m}$ InAs/InP (100) quantum-dot amplifiers," Optical Quantum Electronics, vol. 41, no. 10, pp. 735-749, 2009.

[12] J. Thomson, H. Summers, P. Hulyer, P. Smowton, and P. Blood, "Determination of single-pass optical gain and internal loss using a multisection device," Appl. Phys. Lett., vol. 75, no. 17, pp. 2527-2529, Oct. 1999.

[13] S. D. McDougall and C. N. Ironside, "Measurements of reverse and forward bias absorption and gain spectra in semiconductor laser material," Electron. Lett., vol. 31, no. 25, pp. 2179-2181, Dec. 1995.

[14] P. Blood et al., "Characterization of semiconductor laser gain media by the segmented contact method," IEEE J. Sel. Topics Quantum Electron., vol. 9, no. 5, pp. 1275-1282, Sep. 2003.

[15] Y.-C. Xin et al., "Optical gain and absorption of quantum dots measured using an alternative segmented contact method," IEEE J. Quantum Electron., vol. 42, no. 7, pp. 725-732, Jul. 2006.

[16] G. H. B. Thompson, G. D. Henshall, J. E. A. Whiteaway, and P. A. Kirkby, "Narrow-beam five-layer $(\mathrm{GaAl}) \mathrm{As} / \mathrm{GaAs}$ heterostructure lasers with low threshold and high peak power," J. Appl. Phys., vol. 47, no. 4, pp. 1501-1514, 1976. [Online]. Available: http://scitation. aip.org/content/aip/journal/jap/47/4/10.1063/1.322816

[17] E. Bitincka, R. Broeke, and M. K. Smit, "Electrical method for on-wafer modal gain characterization of semiconductor optical amplifiers," in Proc. 17th Eur. Conf. Int. Opt., Nice, France, Jun. 2014.

[18] J. Verdeyen, Laser Electronics, 3rd ed. Englewood Cliffs, NJ, USA: Prentice-Hall, p. 234, 1995.

[19] Y.-C. Xin et al., "Determination of optical gain and absorption of quantum dots with an improved segmented contact method," in Physics and Simulation of Optoelectronic Devices XIII, M. Osinski, F. Henneberger, and H. Amano, Eds. Bellingham, WA, USA: SPIE, Apr. 2005, pp. 49-59.

[20] L. Negro, P. Bettotti, M. Cazzanelli, D. Pacifici, and L. Pavesi, "Applicability conditions and experimental analysis of the variable stripe length method for gain measurements," Opt. Commun., vol. 229, no. 1, pp. 337-348, 2004. [Online]. Available: http://www. sciencedirect.com/science/article/pii/S0030401803021862

[21] X. Leijtens, "JePPIX: the platform for InP-based photonics," IET Optoelectronics, vol. 5, no. 5, pp. 202-206, 2011.

[22] M. Newville, T. Stensitzki, D. B. Allen, and A. Ingargiola, "LMFIT: Nonlinear least-square minimization and curve-fitting for python,” Sep. 2014. [Online]. Available: https://doi.org/10.5281/zenodo.11813 
Dzmitry Pustakhod received the M.Sc. degree in physics in 2006 from the Belarusian State University, Minsk, Belarus, and he is currently working toward the Ph.D. degree in the Photonic Integration (PhI) group, Department of Electrical Engineering, Eindhoven University of Technology, Eindhoven, The Netherlands. His research interests include the development of process control modules for photonic integration technology, which are used for assessment of the fabrication process and measurement of the performance parameters of the building blocks.

Kevin Williams received the B.Eng. degree in electronic engineering from the University of Sheffield, Sheffield, U.K., and the Ph.D. degree in physics from the University of Bath, Bath, U.K., in 1995. His research interests include the area of integrated photonic circuits. He is the Chair of the Photonic Integration research group at Eindhoven University of Technology, Eindhoven, The Netherlands. He was awarded a Royal Society University research fellowship at the University of Bristol, Bristol, U.K, in 1996. He moved to the University of Cambridge, Cambridge, U.K., in 2001 and was elected a Fellow at Churchill College. In 2006, he was awarded a European Commission Marie Curie Chair at the Eindhoven University of Technology, The Netherlands. In 2011, he received the Vici Award from the Netherlands Organization for Scientific Research (NWO).
Xaveer Leijtens (M'00-SM'13) studied physics at the University of Amsterdam. In 1993, he joined the photonic integrated circuits group at TU Delft. In 2000 he moved to the COBRA research center at TU Eindhoven where he is associate professor in the photonic integration group. His interest focuses on generic photonic integration and he is leading the design effort within the Joint European InP platform, JePPIX.eu. He is a member of the board of the IEEE Photonics Society Benelux chapter. He was an Associate Editor of IEEE Photonics Technology Letters (2007-2011). He (co)authored more than 350 scientific papers and conference contributions. 